\title{
MULTIPLE SOLUTIONS FOR A SEMILINEAR ELLIPTIC EQUATION
}

\author{
MANUEL A. DEL PINO AND PATRICIO L. FELMER
}

ABSTRACT. Let $\Omega$ be a bounded, smooth domain in $\mathbb{R}^{N}, N \geq 1$. We consider the problem of finding nontrivial solutions to the elliptic boundary value problem

$$
\begin{aligned}
\Delta u+\lambda u & =h(x)|u|^{p-1} u \text { in } \Omega, \\
u & =0 \text { on } \partial \Omega,
\end{aligned}
$$

where $h \geq 0, h \not \equiv 0$ is Hölder continuous on $\bar{\Omega}$ and $p>1, \lambda$ are constants.

Let $\Omega_{0}$ denote the interior of the set where $h$ vanishes in $\Omega$. We assume $h>0$ a.e. on $\Omega \backslash \Omega_{0}$ and consider the eigenvalues $\lambda_{i}(\Omega)$ and $\lambda_{i}\left(\Omega_{0}\right)$ of the Dirichlet problem in $\Omega$ and $\Omega_{0}$ respectively. We prove that no nontrivial solution of the equation exists if $\lambda$ satisfies, for some $k \geq 1$,

$$
\lambda_{k}\left(\Omega_{0}\right) \leq \lambda \leq \lambda_{k+1}(\Omega) .
$$

On the other hand, if, for some nonnegative integers $s, k$ with $s \geq k+1, \lambda$ satisfies

$$
\lambda_{s}(\Omega)<\lambda<\lambda_{k+1}\left(\Omega_{0}\right),
$$

then the equation above possesses at least $s-k$ pairs of nontrivial solutions.

For the proof of these results we use a variational approach. In particular, the existence result takes advantage of the even character of the associated functional.

\section{INTRODUCTION}

Let $\Omega$ be a bounded, smooth domain in $\mathbb{R}^{N}, N \geq 1$. We consider the problem of finding nontrivial solutions to the elliptic boundary value problem

$$
\begin{aligned}
\Delta u+\lambda u & =h(x)|u|^{p-1} u \quad \text { in } \Omega, \\
u & =0 \text { on } \partial \Omega,
\end{aligned}
$$

where $h$ is a nonnegative, not identically zero Hölder continuous function defined on $\bar{\Omega}$ and $p>1, \lambda$ are constants.

Ouyang [5] studied existence and uniqueness of positive solutions to problem $(0.1)-(0.2)$ and also of its corresponding analogue on a compact Riemannian manifold. That paper mainly focused on studying the effect of vanishing of the

Received by the editors October 13, 1993 and, in revised form, October 24, 1994.

1991 Mathematics Subject Classification. Primary 35J25, 35J20.

Key words and phrases. Variational method, multiplicity, uniqueness, blow up of solutions.

The work of the first author was partially supported by NSF grant DMS-9100383.

The work of the second author has been partially supported by FONDECYT under grant No $1212-91$. 
function $h$, a question originally raised by Kazdan and Warner [4] who considered the problem on a compact manifold and proved, among other results, that for strictly positive $h$ there is a unique positive solution for any $\lambda>0$. Similarly, a unique positive solution to (0.1)-(0.2) exists for any $\lambda>\lambda_{1}(\Omega)$, where $\lambda_{1}(\Omega)$ denotes the first Dirichlet eigenvalue of the Laplacian in $\Omega$. An integration by parts easily yields that this condition is also necessary for existence of a nontrivial solution to $(0.1)-(0.2)$.

Let $\Omega_{0}$ denote the interior of the set where $h$ vanishes in $\Omega$. We will assume henceforth that $\Omega_{0}$ is nonempty and that $h>0$ a.e. on $\Omega \backslash \Omega_{0}$. Then the following result holds; see [5] and also [3] for an alternative proof.

Theorem 0.1. There exists a unique positive solution $u_{\lambda}$ to problem $(0.1)-(0.2)$ if $\lambda_{1}(\Omega)<\lambda<\lambda_{1}\left(\Omega_{0}\right)$. Moreover,

$$
\lim _{\lambda \rightarrow \lambda_{1}\left(\Omega_{0}\right)}\left\|u_{\lambda}\right\|_{L^{2}}=+\infty
$$

and no positive solution exists in case that $\lambda \geq \lambda_{1}\left(\Omega_{0}\right)$.

Ouyang actually proves that the set of all pairs $(\lambda, u)$ with $u$ a positive solution of $(0.1)-(0.2)$ is constituted exactly by the first bifurcation branch starting at $\left(\lambda_{1}(\Omega), 0\right)$ and that this branch continues to exist until $\lambda$ reaches $\lambda_{1}\left(\Omega_{0}\right)$ where it blows up. We should remark that a very different behavior of this branch takes place in case that $h$ changes sign (see [6]).

Now, it follows from standard bifurcation theory that a branch of nontrivial solutions of (0.1)-(0.2) starts at each eigenvalue of the Laplacian, provided that this eigenvalue is e.g. simple. Thus, the natural question arises: Will branches associated with higher eigenvalues exhibit a similar behavior to that of the first one? This paper is mainly motivated by this question. Giving a complete answer may be very hard. However, our first result, Theorem 0.2 below, partially recovers the picture of the first branch: it shows, in particular, that if the Dirichlet eigenvalues of both $\Omega$ and $\Omega_{0}$ are simple, then for each positive integer $k$ there exists a family of pairs $\left(\lambda, \pm u_{\lambda}^{k}\right)$ with $u_{\lambda}^{k}$ a nontrivial solution of (0.1)-(0.2), defined for $\lambda \in\left(\lambda_{k}(\Omega), \lambda_{k}\left(\Omega_{0}\right)\right)$, with the property that $\left\|u_{\lambda}^{k}\right\|_{L^{2}}$ blows up as $\lambda$ approaches $\lambda_{k}\left(\Omega_{0}\right)$. Moreover, these "branches" do not intersect each other in the sense that, if $\lambda$ belongs to $j$ intervals of the form $\left(\lambda_{k}(\Omega), \lambda_{k}\left(\Omega_{0}\right)\right)$ then at least $j$ pairs of nontrivial solutions exist.

To state our first result in the general case, we consider the sequences of Dirichlet eigenvalues for $\Omega$ and $\Omega_{0}$,

$$
\begin{gathered}
\lambda_{1}(\Omega)<\lambda_{2}(\Omega) \leq \lambda_{3}(\Omega) \leq \lambda_{4}(\Omega) \leq \cdots, \\
\lambda_{1}\left(\Omega_{0}\right)<\lambda_{2}\left(\Omega_{0}\right) \leq \lambda_{3}\left(\Omega_{0}\right) \leq \cdots
\end{gathered}
$$

where a given eigenvalue in the above sequences appears as many times as its multiplicity indicates. If $\Omega_{0}$ is a general open subset of $\Omega$, with no regularity assumed on its boundary, its Dirichlet eigenvalues can be naturally defined considering the closed subspace $H_{*}^{1}\left(\Omega_{0}\right)$ of $H_{0}^{1}(\Omega)$ given by

$$
H_{*}^{1}\left(\Omega_{0}\right)=\left\{u \in H_{0}^{1}(\Omega) \mid u=0 \text { a.e. on } \Omega \backslash \Omega_{0}\right\} \text {. }
$$

It is well known that $H_{*}^{1}\left(\Omega_{0}\right)=H_{0}^{1}\left(\Omega_{0}\right)$ if $\Omega_{0}$ has, for example, a Lipschitz continuous boundary. We define the sequence of Dirichlet eigenvalues of $\Omega_{0}$ as those of the problem

$$
u=\lambda T u, \quad u \in H_{*}^{1}\left(\Omega_{0}\right),
$$


where $T$ is the compact, selfadjoint operator so that $v=T f$ is the unique element of $H_{*}^{1}\left(\Omega_{0}\right)$ satisfying

$$
\int_{\Omega_{0}} \nabla v \nabla \phi=\int_{\Omega_{0}} f \phi \quad \forall \phi \in H_{*}^{1}\left(\Omega_{0}\right)
$$

Note that the variational characterization of the eigenvalues yields

$$
\lambda_{k}(\Omega) \leq \lambda_{k}\left(\Omega_{0}\right) \quad \forall k \geq 1 .
$$

Our first result is the following

Theorem 0.2. Assume that there exist nonnegative integers $s, k$ with $s \geq k+1$ such that $\lambda$ satisfies

$$
\lambda_{s}(\Omega)<\lambda<\lambda_{k+1}\left(\Omega_{0}\right) .
$$

Then there exist at least $(s-k)$ distinct pairs of nontrivial solutions $\pm u_{\lambda}^{k+1}$, $\pm u_{\lambda}^{k+2}, \ldots, \pm u_{\lambda}^{s}$ to $(0.1)-(0.2)$. Moreover, if $q$ is the multiplicity of $\lambda_{k+1}\left(\Omega_{0}\right)$, then the above families can be selected in such a way that

(i)

$$
\lim _{\lambda \rightarrow \lambda_{k+1}\left(\Omega_{0}\right)}\left\|u_{\lambda}^{l}\right\|_{L^{2}}=+\infty, \quad k+1 \leq l \leq \min \{s, k+q\},
$$

and

(ii) $\left\|u_{\lambda}^{l}\right\|_{L^{2}}$ remains uniformly bounded on $\lambda$ satisfying (0.4) for all $l$ satisfying $k+q<l \leq s$, in case that $q<s-k$.

Observe that among the assumptions of the above result we may also include, without loss of generality,

$$
\lambda_{s+1}(\Omega)>\lambda_{s}(\Omega) \text { and } \lambda_{k}\left(\Omega_{0}\right)<\lambda_{k+1}\left(\Omega_{0}\right),
$$

in which case the number of families predicted by the theorem becomes maximal.

Let us notice that if the eigenvalues of $\Omega_{0}$ are simple, this result provides, for each $k \geq 1$, a family of pairs of nontrivial solutions $u_{\lambda}^{l}$, defined for $\lambda_{k}(\Omega)<$ $\lambda<\lambda_{k}\left(\Omega_{0}\right)$, which blows up in $L^{2}$-norm as $\lambda$ approaches $\lambda_{k}\left(\Omega_{0}\right)$.

It is natural to ask what happens if $\lambda$ does not belong to any interval of the real line covered by the above theorem. It is maybe a little surprising that a full answer is possible in this situation: no nontrivial solution of $(0.1)-(0.2)$ exists.

Theorem 0.3. Assume that $\lambda$ satisfies, for some $k \geq 1$,

$$
\lambda_{k}\left(\Omega_{0}\right) \leq \lambda \leq \lambda_{k+1}(\Omega) .
$$

Then $(0.1)-(0.2)$ possesses only the solution $u \equiv 0$.

The proofs of Theorems 0.2 and 0.3 are based on variational arguments applied to the functional

$$
J_{\lambda}(u)=\frac{1}{2} \int_{\Omega}|\nabla u|^{2}-\lambda u^{2}+\frac{1}{p+1} \int_{\Omega} h|u|^{p+1}
$$

defined on the Banach space

$$
X=\left\{\left.u \in H_{0}^{1}(\Omega)\left|\int_{\Omega} h\right| u\right|^{p+1}<+\infty\right\}
$$


endowed with norm

$$
\|u\|_{X}=\left(\int_{\Omega}|\nabla u|^{2}\right)^{1 / 2}+\left(\int_{\Omega} h|u|^{p+1}\right)^{\frac{1}{p+1}} .
$$

It is standard to check that $J_{\lambda}$ is of class $C^{1}$ on $X$ and that its critical points correspond exactly to the solutions of $(0.1)-(0.2)$. We should remark that the positive solutions correspond precisely to global minimizers of this functional as established in [3]. If $\lambda \geq \lambda_{1}\left(\Omega_{0}\right)$ the functional becomes unbounded below. We will take advantage of the even character of the functional $J_{\lambda}$ in order to establish existence of multiple critical points under the assumptions of Theorem 0.2. Properties (i) and (ii) will follow from appropriate min-max characterizations of the associated critical values. Actually, these characterizations give that $J_{\lambda}\left(u_{\lambda}^{l}\right) \rightarrow-\infty$ as $\lambda$ approaches $\lambda_{k+1}\left(\Omega_{0}\right)$, in case (i) and remains locally uniformly bounded for $\lambda$ in the range given by (ii).

On the other hand, the result of Theorem 0.3 actually holds true for a much larger class of nonlinearities. In particular, no symmetry is required. See Remark 1.1 below.

The rest of this paper will be devoted to the proof of the above results. Its outline is as follows. In $\S 1$ we prove the nonexistence result of Theorem 0.3 . In $\S 2$ we show that $J_{\lambda}$ satisfies the Palais-Smale condition for any value of $\lambda$, a necessary step for the proof of Theorem 0.2 , which we carry out in $\S 3$.

In what remains of this paper $X$ will always denote the space given by $(0.6)$ with its associated norm. In the space $H_{0}^{1}(\Omega)$ we consider the usual inner product

$$
\langle\psi, \phi\rangle=\int_{\Omega} \nabla \psi \nabla \phi, \quad \psi, \phi \in H_{0}^{1}(\Omega),
$$

and denote by $\|\cdot\|_{H_{0}^{1}}$ its associated norm. Correspondingly, we denote by $\|\cdot\|_{L^{2}}$ the usual norm in $L^{2}(\Omega)$.

We also choose orthogonal bases of $H_{0}^{1}(\Omega)$ and $H_{*}^{1}\left(\Omega_{0}\right)$ respectively denoted by $\left\{\psi_{i}\right\}_{i=1}^{\infty}$ and $\left\{\phi_{i}\right\}_{i=1}^{\infty}$ such that $\psi_{i}$ is an eigenfunction associated to $\lambda_{i}(\Omega)$ and $\phi_{i}$ an eigenfunction for $\lambda_{i}\left(\Omega_{0}\right)$, normalized so that $\left\|\phi_{i}\right\|_{L^{2}}=\left\|\psi_{i}\right\|_{L^{2}}=1$.

\section{Proof of Theorem 0.3}

In this section we shall prove that if for some $k \geq 1$ one has

$$
\lambda_{k}\left(\Omega_{0}\right) \leq \lambda \leq \lambda_{k+1}(\Omega),
$$

then no nontrivial solution of $(0.1)-(0.2)$ exists. We begin with a preliminary result.

Lemma 1.1. For any $\varepsilon>0$ there exist functions:

(a) $\psi_{1}^{\varepsilon}, \psi_{2}^{\varepsilon}, \ldots, \psi_{k}^{\varepsilon} \in H_{0}^{1}(\Omega)$ such that $\left\|\psi_{i}-\psi_{i}^{\varepsilon}\right\|_{H_{0}^{1}}<\varepsilon$ for $i=1, \ldots, k$ and

$$
\operatorname{span}\left\{\phi_{1}, \ldots, \phi_{k}\right\} \cap \operatorname{span}\left\{\psi_{1}^{\varepsilon}, \ldots \psi_{k}^{\varepsilon}\right\}^{\perp}=\{0\} \text {, and }
$$

(b) $\phi_{1}^{\varepsilon}, \phi_{2}^{\varepsilon}, \ldots, \phi_{k}^{\varepsilon} \in H_{*}^{1}\left(\Omega_{0}\right)$ such that $\left\|\phi_{i}-\phi_{i}^{\varepsilon}\right\|_{H_{0}^{\prime}}<\varepsilon$ for $i=1, \ldots, k$ and

$$
\operatorname{span}\left\{\phi_{1}^{\varepsilon}, \ldots, \phi_{k}^{\varepsilon}\right\} \cap \operatorname{span}\left\{\psi_{1}, \ldots, \psi_{k}\right\}^{\perp}=\{0\} .
$$

Here, $\perp$ denotes the orthogonal in $H_{0}^{1}(\Omega)$. 
Proof. First we prove (a). We need to choose functions $\eta_{1}^{\varepsilon}, \ldots, \eta_{k}^{\varepsilon}$ with $\left\|\eta_{i}^{\varepsilon}\right\|_{H_{0}^{1}}<\varepsilon$ such that if we set $\psi_{i}^{\varepsilon}=\psi_{i}+\eta_{i}^{\varepsilon}$, then the matrix

$$
A_{\varepsilon}=\left\{\left\langle\psi_{i}^{\varepsilon}, \phi_{j}\right\rangle\right\}_{i, j}
$$

is invertible. Observe that we can find numbers $\lambda_{i j}$ with $\sum_{i, j} \lambda_{i j}^{2}$ arbitrarily small, so that the matrix with coefficients $\left\langle\psi_{i}, \phi_{j}\right\rangle+\lambda_{i j}$ is invertible. Let us set $\eta_{i}^{\varepsilon}=\sum_{j=1}^{k} \lambda_{i j} \phi_{j}$. For appropriately chosen $\lambda_{i j}$, the $\eta_{i}^{\varepsilon}$ defined in this way will clearly satisfy the desired properties.

For the proof of part (b), we need to find functions $\eta_{1}^{\varepsilon}, \ldots, \eta_{k}^{\varepsilon} \in H_{*}^{1}\left(\Omega_{0}\right)$ with $\left\|\eta_{i}^{\varepsilon}\right\|_{H_{0}^{1}}<\varepsilon$ such that the matrix with coefficients $\left\langle\psi_{i}, \phi_{j}+\eta_{j}^{\varepsilon}\right\rangle$ is invertible. Note first that the matrix with coefficients $\int_{\Omega_{0}} \psi_{i} \psi_{j}$ is invertible. Indeed, this is a consequence of the fact that the functions $\psi_{1}, \ldots, \psi_{k}$ are linearly independent on $\Omega_{0}$, as follows from the unique continuation property for a linear combination of them. Now choose functions $\tilde{\psi}_{1}, \ldots, \tilde{\psi}_{k} \in C_{0}^{\infty}\left(\Omega_{0}\right)$ such that the matrix with coefficients $\int_{\Omega_{0}} \psi_{i} \tilde{\psi}_{j}$ is still invertible. Finally, as in part (a), $\eta_{i}^{\varepsilon}$ defined as some linear combination of the $\tilde{\psi}_{j}$ 's with sufficiently small coefficients will satisfy the desired requirement.

Proof of Theorem 0.3. First we consider the case

$$
\lambda_{k}\left(\Omega_{0}\right)<\lambda<\lambda_{k+1}(\Omega) .
$$

For a small number $\varepsilon$ to be chosen, we consider functions $\psi_{1}^{\varepsilon}, \ldots, \psi_{k}^{\varepsilon}$ as in Lemma 1.1, part (a). Let us set $V=\operatorname{span}\left\{\phi_{1}, \ldots, \phi_{k}\right\}$ and

$$
Y=\left\{y \in X \mid\left\langle y, \psi_{i}^{e}\right\rangle=0, i=1, \ldots, k\right\} \text {. }
$$

For $v=\sum_{i=1}^{k} t_{i} \phi_{i}$ and $y \in Y$ we see that

$$
J_{\lambda}(v+y)=J_{\lambda}(y)+\sum_{i=1}^{k} \frac{t_{i}^{2}}{2}\left(\lambda_{i}\left(\Omega_{0}\right)-\lambda\right)+\sum_{i=1}^{k} t_{i} \int_{\Omega}\left(\nabla \phi_{i} \nabla y-\lambda \phi_{i} y\right) .
$$

Let $\tilde{u}=\tilde{v}+\tilde{y}$ be a critical point of $J_{\lambda}$. We will show that $\tilde{u} \equiv 0$. To do this, we see that, for each fixed $y \in Y$, the functional $v \mapsto J_{\lambda}(v+y)$ has a unique critical point $v=w(y)=\sum_{i=1}^{k} t_{i}(y) \phi_{i}$ where

$$
t_{i}(y)=-\frac{\int_{\Omega} \nabla \phi_{i} \nabla y-\lambda \phi_{i} y}{\lambda_{i}\left(\Omega_{0}\right)-\lambda}, \quad i=1, \ldots, k .
$$

Thus, we must have $\tilde{v}=w(\tilde{y})$. Moreover, $\tilde{y}$ must be a critical point of the functional

$$
y \mapsto J_{\lambda}(w(y)+y)=J_{\lambda}(y)+\frac{1}{2} \sum_{i=1}^{k} \frac{\left(\int_{\Omega} \nabla \phi_{i} \nabla y-\lambda \phi_{i} y\right)^{2}}{\lambda-\lambda_{i}\left(\Omega_{0}\right)} .
$$

We will check that the above functional is strictly convex if $\varepsilon$ is chosen sufficiently small. To do this, it clearly suffices to show that the quadratic functional

$$
I_{\lambda}(y)=\int_{\Omega}|\nabla y|^{2}-\lambda \int_{\Omega}|y|^{2}
$$


is strictly convex on $Y$, which is equivalent to verifying that $I_{\lambda}(y)>0$ for $y \neq 0$. Let $\bar{y}=\sum_{i=1}^{k}\left\langle y, \psi_{i}\right\rangle \psi_{i}, x=y-\bar{y}$. Then $I_{\lambda}(y)=I_{\lambda}(\bar{y})+I_{\lambda}(x)$, and since $\lambda<\lambda_{k+1}(\Omega)$, we have

$$
I_{\lambda}(y) \geq\left(1-\frac{\lambda}{\lambda_{k+1}(\Omega)}\right)\|x\|_{H_{0}^{1}}^{2}+\left(1-\frac{\lambda}{\lambda_{1}(\Omega)}\right)\|\bar{y}\|_{H_{0}^{\prime}}^{2} .
$$

But

$$
\|\bar{y}\|_{H_{0}^{1}}^{2}=\sum_{i=1}^{k}\left|\left\langle y, \psi_{i}\right\rangle\right|^{2}=\sum_{i=1}^{k}\left|\left\langle y, \psi_{i}-\psi_{i}^{\varepsilon}\right\rangle\right|^{2} \leq k \varepsilon^{2}\|y\|_{H_{0}^{1}}^{2}
$$

and hence

$$
\|x\|_{H_{0}^{1}}^{2}=\|y\|_{H_{0}^{1}}^{2}-\|\bar{y}\|_{H_{0}^{1}}^{2} \geq\left(1-k \varepsilon^{2}\right)\|y\|_{H_{0}^{1}}^{2} .
$$

Combining (1.7), (1.8) and (1.9) we obtain, for $\varepsilon$ sufficiently small,

$$
I_{\lambda}(y)>0 \text { if } y \neq 0 \text {. }
$$

It follows that the functional (1.5) has $y=0$ as its unique critical point, so that $\tilde{x}=0$ and hence $u=0$, as desired.

Next let us assume that $\lambda=\lambda_{k}\left(\Omega_{0}\right)$ and let $q$ be the multiplicity of $\lambda_{k}\left(\Omega_{0}\right)$. In this case, for each $y \in Y$, the functional $v \mapsto J_{\lambda}(y+v)$ has a critial point if and only if

$$
\int_{\Omega} \nabla \phi_{l} \nabla y-\lambda \int_{\Omega} \phi_{l} y=0, \quad k \leq l \leq k-q+1 .
$$

And in such case the critical points are given by the $q$-parameter family

$$
v_{s}(y)=\sum_{i=1}^{k-q} t_{i}(y) \phi_{i}+\sum_{i=k-q+1}^{k} s_{i} \phi_{i}, \quad s=\left(s_{k-q+1}, \ldots, s_{k}\right) \in \mathbb{R}^{q}
$$

where $t_{i}(y)$ is as in (1.4). Then if $\tilde{u}=\tilde{v}+\tilde{y}$ is a critical point of $J_{\lambda}$, then $\tilde{v}=v_{\tilde{s}}(\tilde{y})$ for some $\tilde{s} \in \mathbb{R}^{q}$, and $\tilde{y}$ must be a critical point of the functional $y \rightarrow J_{\lambda}\left(y+v_{\tilde{s}}(y)\right)$. But, similarly as in the previous case, an appropriate choice of $\varepsilon$ yields that $y=0$ is the only critical point of this functional. Thus, $\tilde{u}=\sum_{i=k-q+1}^{k} \tilde{s}_{i} \phi_{i}$ is a critical point of $J_{\lambda}$ and hence satisfies

$$
\Delta \tilde{u}+\lambda_{k}\left(\Omega_{0}\right) \tilde{u}=0 \text { in } \Omega \text {. }
$$

The unique continuation property of the solutions of (1.10) yields that $\tilde{u}=0$, as desired.

It only remains to consider the case $\lambda=\lambda_{k+1}(\Omega)$. Let $\phi_{1}^{\varepsilon}, \ldots, \phi_{k}^{\varepsilon}$ be as in Lemma 1.1, part (b) and consider the decomposition $X=V \oplus Y$ where now $V=\operatorname{span}\left\{\phi_{1}^{\varepsilon}, \ldots, \phi_{k}^{\varepsilon}\right\}$, and

$$
Y=\left\{y \in X \mid\left\langle y, \psi_{i}\right\rangle=0, i=1, \ldots, k\right\} .
$$


For $v=\sum_{i=1}^{k} t_{i} \phi_{i}^{\varepsilon}$ and $y \in Y$ we now have that

$$
\begin{aligned}
J_{\lambda}(v+y)= & J_{\lambda}(y)+\sum_{i=1}^{k} \frac{t_{i}^{2}}{2}\left(\lambda_{i}\left(\Omega_{0}\right)-\lambda\right) \\
& +\sum_{i=1}^{k} t_{i}\left(\int_{\Omega} \nabla \phi_{i}^{\varepsilon} \nabla y-\lambda \phi_{i}^{\varepsilon} y\right) \\
& +\frac{1}{2} \sum_{i=1}^{k} \sum_{j=1}^{k} t_{i} t_{j} o_{i j}(\varepsilon)
\end{aligned}
$$

where

$$
o_{i j}(\varepsilon)=\int_{\Omega} \nabla\left(\phi_{i}+\eta_{i}^{\varepsilon}\right) \nabla \eta_{j}^{\varepsilon}-\lambda\left(\phi_{i}+\eta_{i}^{\varepsilon}\right) \eta_{j}^{\varepsilon}
$$

with $\eta_{i}^{\varepsilon}=\phi_{i}-\phi_{i}^{\varepsilon}$. From the choice of the functions $\phi_{i}^{\varepsilon}$ in Lemma 1.1, part (b) we certainly have that $\lim _{\varepsilon \rightarrow 0} o_{i j}(\varepsilon)=0$ for all $1 \leq i, j \leq k$. Recalling that, for $1 \leq i \leq k, \lambda_{i}\left(\Omega_{0}\right)-\lambda<0$ we see that for $\varepsilon>0$ sufficiently small, as in the previous cases, $\tilde{u}=\tilde{v}+\tilde{y}$ will be a critical point of $J_{\lambda}$ if and only if $\tilde{v}=w(\tilde{y})=\sum_{i=1}^{k} t_{i}(\tilde{y}) \phi_{i}^{\varepsilon}$ with $t(y)=\left(t_{1}(y), \ldots, t_{k}(y)\right)$ given by

$$
t(y)=-(D+O(\varepsilon))^{-1} b(y)
$$

where $D=\operatorname{diag}\left(\lambda_{i}\left(\Omega_{0}\right)-\lambda\right), O(\varepsilon)=\left(o_{i j}(\varepsilon)\right)_{i j}$ and $b(y)=\left(b_{1}(y), \ldots, b_{k}(y)\right)$ is the vector with components

$$
b_{i}(y)=\int_{\Omega} \nabla \phi_{i}^{\varepsilon} \nabla y-\lambda \phi_{i}^{\varepsilon} y .
$$

Moreover, $\tilde{y}$ is a critical point of the functional

$$
y \mapsto J_{\lambda}(w(y)+y)=J_{\lambda}(y)-\frac{1}{2} b(y)^{\tau}(D+O(\varepsilon))^{-1} b(y) .
$$

But this functional is convex since $\int_{\Omega}|\nabla y|^{2}-\lambda \int_{\Omega}|y|^{2} \geq 0$ and $D+O(\varepsilon)$ is negative definite for $\varepsilon$ small enough. Thus, its critical points are all minima and then for $\tilde{y}$, a minimum of (1.13), we have

$$
\int_{\Omega}|\nabla \tilde{y}|^{2}-\lambda \int_{\Omega}|\tilde{y}|^{2}=0 \text {. }
$$

Hence $\tilde{y}=\sum_{i=1}^{q} \tilde{t}_{i} \psi_{k+i}$ for some $\tilde{t}=\left(t_{1}, \ldots, t_{q}\right) \in \mathbb{R}^{q}$, where $q$ is the multiplicity of $\lambda_{k+1}(\Omega)$. Also, at the minimum $\tilde{y}$ we have

$$
\frac{1}{p+1} \int_{\Omega} h|\tilde{y}|^{p+1}=0
$$

hence $\tilde{y}$ has its support contained in $\Omega_{0}$. The unique continuation property of the eigenfunctions $\left\{\psi_{i}\right\}$ thus yields $\tilde{y}=0$, and therefore $\tilde{u} \equiv 0$ as desired. This concludes the proof.

Remark 1.1. The preceding arguments actually apply to the more general problem

$$
\begin{aligned}
\Delta u+\lambda u & =h(x) f(u) \text { in } \Omega, \\
u & =0 \text { on } \partial \Omega,
\end{aligned}
$$


where we assume that $f$ is locally Hölder continuous, nondecreasing and such that $u f(u)>0$ for $u \neq 0$. In fact, exactly the same proof yields that if

$$
\lambda_{k}\left(\Omega_{0}\right) \leq \lambda \leq \lambda_{k+1}(\Omega),
$$

then (1.14)-(1.15) possesses only the solution $u \equiv 0$.

\section{The Palais-Smale condition}

The proof of Theorem 0.2 involves variational arguments applied to the functional $J_{\lambda}$ defined on the space $X$ given by $(0.6)$ as

$$
J_{\lambda}(u)=\frac{1}{2} \int_{\Omega}|\nabla u|^{2}-\lambda u^{2}+\frac{1}{p+1} \int_{\Omega} h|u|^{p+1} .
$$

In this section we will show that the Palais-Smale condition holds for $J_{\lambda}$ for any value of $\lambda$.

Lemma 2.1. $J_{\lambda}$ satisfies the Palais-Smale condition for any $\lambda$; that is, any sequence $\left\{u_{n}\right\} \subset X$ such that $J_{\lambda}\left(u_{n}\right)$ is bounded and $J_{\lambda}^{\prime}\left(u_{n}\right) \rightarrow 0$ in $X^{*}$ possesses a convergent subsequence in $X$.

Proof. As a first step we show that the sequence $\left\{u_{n}\right\}$ as in the statement of the lemma is bounded in $X$. Assume $J_{\lambda}\left(u_{n}\right) \rightarrow C \in \mathbb{R}$ or

$$
\frac{1}{2} \int_{\Omega}\left|\nabla u_{n}\right|^{2}-\lambda u_{n}^{2}+\frac{1}{p+1} \int_{\Omega} h\left|u_{n}\right|^{p+1}=C+o(1) .
$$

We also have

$$
\int_{\Omega} \nabla u_{n} \nabla \phi-\lambda \int_{\Omega} u_{n} \phi+\int_{\Omega} h\left|u_{n}\right|^{p-1} u_{n} \phi=o(1)\|\phi\|_{X}
$$

for all $\phi \in X$ where $o(1) \rightarrow 0$ uniformly on $\phi$.

Let us assume, by contradiction, $\left\|u_{n}\right\|_{X} \rightarrow+\infty$. Then, from (2.1) we see that, for some $c>0$,

$$
\left\|u_{n}\right\|_{X} \leq c\left\|u_{n}\right\|_{L^{2}}
$$

for large $n$.

Let us set $\hat{u}_{n}=u_{n} /\left\|u_{n}\right\|_{L^{2}}$. Then, from (2.3), $\hat{u}_{n}$ is bounded in $X$ and hence, we may assume $\hat{u}_{n} \rightarrow \hat{u}$, weakly in $H_{0}^{1}(\Omega)$ and strongly in $L^{2}(\Omega)$ to some $\hat{u} \in X$ such that $\|\hat{u}\|_{L^{2}}=1$.

Choosing $\phi=u_{n}$ in (2.2), combining with (2.1) and using (2.3) we obtain

$$
\left(\frac{1}{2}-\frac{1}{p+1}\right) \int_{\Omega} h\left|u_{n}\right|^{p+1}=o(1)\left\|u_{n}\right\|_{L^{2}} \text {. }
$$

It follows from (2.4) and Fatou's Lemma that

$$
\int_{\Omega} h|\hat{u}|^{p+1}=0
$$

and hence $\hat{u}=0$ a.e. on $\Omega \backslash \Omega_{0}$, that is, $\hat{u} \in H_{*}^{1}\left(\Omega_{0}\right)$. Fix $\phi \in C_{0}^{\infty}(\Omega)$, and observe that

$$
\left.\left|\int_{\Omega} h\right| u_{n}\right|^{p-1} u_{n} \phi \mid \leq c\|\phi\|_{L^{\infty}}\left(\int_{\Omega} h\left|u_{n}\right|^{p+1}\right)^{p /(p+1)} .
$$


It follows from (2.2), (2.5) and (2.6) that

$$
\int_{\Omega} \nabla \hat{u} \nabla \phi-\lambda \int_{\Omega} \hat{u} \phi=0 .
$$

Hence $\hat{u}$ is regular and satisfies

$$
\Delta \hat{u}+\lambda \hat{u}=0 \text { in } \Omega .
$$

But $\hat{u} \equiv 0$ on $\Omega \backslash \Omega_{0}$; thus the unique continuation property of the solutions of (2.7) implies $\hat{u} \equiv 0$ on $\Omega$. This is a contradiction since $\|\hat{u}\|_{L^{2}}=1$; then $\left\{u_{n}\right\}$ is bounded in $H_{0}^{1}(\Omega)$. Thus we can assume that the sequence $\left\{u_{n}\right\}$ is weakly convergent in $H_{0}^{1}(\Omega)$, strongly in $L^{2}(\Omega)$, and also a.e., to some $u \in H_{0}^{1}(\Omega)$. In order to show that the sequence converges in $X$ it suffices to show that $\left\|u_{n}\right\|_{X}$ converges to $\|u\|_{X}$.

From (2.2) with $\phi=u_{n}$ we obtain

$$
\lim _{n \rightarrow \infty} \int_{\Omega}\left|\nabla u_{n}\right|^{2}+h\left|u_{n}\right|^{p+1}=\lambda \int_{\Omega} u^{2}
$$

Next, writing $J_{\lambda}=I+K$ where

$$
I(u)=\frac{1}{2} \int_{\Omega}|\nabla u|^{2}+\frac{1}{p+1} \int_{\Omega} h|u|^{p+1}
$$

and using the convexity of $I$ we see that for every $v \in X$

$$
\left(I^{\prime}\left(u_{n}\right)-I^{\prime}(v)\right)\left(u_{n}-v\right)=\left(-K^{\prime}\left(u_{n}\right)+o(1)-I^{\prime}(v)\right)\left(u_{n}-v\right) \geq 0 .
$$

Choosing $v$ appropriately and letting $n \rightarrow \infty$ we conclude that

$$
\int_{\Omega}|\nabla u|^{2}+\int_{\Omega} h|u|^{p+1}=\lambda \int_{\Omega} u^{2}
$$

On the other hand, we have

$$
\int_{\Omega}|\nabla u|^{2} \leq \liminf _{n \rightarrow \infty} \int_{\Omega}\left|\nabla u_{n}\right|^{2}
$$

and

$$
\int_{\Omega} h|u|^{p+1} \leq \liminf _{n \rightarrow \infty} \int_{\Omega} h\left|u_{n}\right|^{p+1} .
$$

From these inequalities and (2.8), (2.9), we finally obtain that $\left\|u_{n}\right\|_{X}$ converges to $\|u\|_{X}$, as desired.

\section{Proof of Theorem 0.2}

In this section we will prove that the functional $J_{\lambda}$ defined in $(0.5)$ possesses at least $s-k$ pairs of critical points when we are under the assumption

$$
\lambda_{s}(\Omega)<\lambda<\lambda_{k+1}\left(\Omega_{0}\right)
$$

with $s \geq k+1$.

For this purpose we will use a variant of a result of Clark [2]; see also Rabinowitz [7]. Actually the result we need is a version of a theorem of Benci, Theorem 0.1 in [1]. For the reader's convenience we recall the notion of the genus, enumerate its main properties and sketch the proof of the critical point theorem. 
We consider the Banach space $X$. We denote by $\mathscr{E}$ the class of closed subsets $A$ of $X \backslash\{0\}$ that are symmetric in the sense that $x \in A$ implies $-x \in A$. If $A \in \mathscr{E}$, then the genus of $A$ is $k$ if there is a continuous odd function

$$
\varphi: A \rightarrow \mathbb{R}^{k} \backslash\{0\}
$$

and $k$ is the smallest integer with this property. If such a $k$ does not exist, we say that the genus of $A$ is $\infty$. We write $\gamma(a)$ for the genus of $A$. In what follows we denote by $N_{\delta}(A)$ the uniform $\delta$-neighborhood of the subset $A$ of $X$, i.e. $N_{\delta}=\{x \in X \mid\|x-A\| \leq \delta\}$.

Proposition 3.1. If $A, B \in \mathscr{E}$, then:

(1) Normalization: If $x \neq 0$, then $\gamma(\{x\} \cup\{-x\})=1$.

(2) Monotonicity: If $\varphi: A \rightarrow B$ is a continuous odd function, then $\gamma(A) \leq$ $\gamma(B)$. In particular if $A \subset B$, then $\gamma(A) \leq \gamma(B)$.

(3) Subadditivity: $\gamma(A \cup B) \leq \gamma(A)+\gamma(B)$.

(4) Continuity: If $A$ is compact, $\gamma(A)<\infty$ and there exists $\delta>0$ so that $N_{\delta}(A) \in \mathscr{E}$ and $\gamma(A)=\gamma\left(N_{\delta}(A)\right)$.

(5) Dimension: If $A \in \mathscr{E}, \mathscr{V}$ is a bounded neighborhood of 0 in $\mathbb{R}^{k}$, and there exists an odd homeomorphism between $A$ and $\partial \mathscr{V}$, then $\gamma(A)=k$.

For a proof of these properties we refer the reader to [7, Chapter 7]. Using these properties the following lemma can be proved (see Lemma 2.6 in [1]).

Lemma 3.1. If $A \in \mathscr{E}$ is compact and $Y$ is a subspace of $X$ of finite codimension, then $\gamma(A \cap Y) \geq \gamma(A)-\operatorname{codim}(Y)$.

Proof. Let $V$ be a vector subspace of $X$ so that $X=V \oplus Y$ and consider $P: X \rightarrow V$ the projection onto $V$. Since $A$ is compact, the continuity property implies that there exists $\delta>0$ so that $\gamma(A \cap Y)=\gamma\left(N_{\delta}(A \cap Y)\right)$. Put $N=$ $N_{\delta}(A \cap Y)$.

Denote by $S$ the codimension of $Y$ and assume that

$$
\gamma(A \cap Y)<\gamma(A)-s .
$$

If we put $A_{1}=A \cap N$ and $A_{2}=\overline{A \backslash N}$, then $0 \notin P\left(A_{2}\right)$ and then, by the monotonicity and dimension properties of the genus,

$$
\gamma\left(A_{2}\right) \leq \gamma\left(P\left(A_{2}\right)\right) \leq s .
$$

On the other hand, using monotonicity and subadditivity we obtain

$$
\gamma(A) \leq \gamma\left(A_{1}\right)+\gamma\left(A_{2}\right) \leq \gamma(N)+\gamma\left(A_{2}\right)<\gamma(A)-s+s,
$$

a contradiction from which the result follows.

Next we formulate the critical point theorem to be used in our specific situation.

Theorem 3.1. Let $X$ be a real Banach space. Let $I: X \rightarrow \mathbb{R}$ be a $C^{1}$ functional satisfying the Palais-Smale condition. Assume further that

(1) $I$ is even and $I(0)=0$.

(2) There is a closed subspace $Y$ of $X$ so that $I$ is bounded below on $Y$.

(3) There is a closed subset $S \in \mathscr{E}$ and a constant $b>0$ so that $I(w) \leq-b$, $\forall w \in S$, and 
(4) If $\mathscr{S}^{h}=h(S) \cap Y$, then $\gamma\left(\mathscr{S}^{h}\right) \geq m$ for all functions $h$ in the class of functions

$$
\Gamma=\{h: S \rightarrow X \backslash\{0\} \mid h \text { is continuous and odd }\} .
$$

Then the functional $I$ possesses at least $m$ pairs of critical points and their critical values have a minimax characterization (see (3.2)).

Proof. Since the proof is rather standard, we will be sketchy. First we define a class of sets. Given $1 \leq l \leq m$ we consider the classes $\mathscr{A}_{l}$ defined as follows: $A \in \mathscr{A}_{l}$ if and only if $A=h(S) \backslash K$ such that $h \in \Gamma, \gamma(K) \leq m-l$ and $K \in \mathscr{E}$ is compact. By the monotonicity property of genus we have that $\mathscr{A}_{m} \subset \mathscr{A}_{m-1} \subset$ $\cdots \subset \mathscr{A}_{1}$.

For $1 \leq l \leq m$ we define the numbers

$$
\dot{c}_{l}=\inf _{A \in \mathscr{A}_{l}} \sup _{u \in A} I(u) \text {. }
$$

Since the classes $\mathscr{A}_{l}$ are ordered, we have $c_{1} \leq c_{2} \leq \cdots \leq c_{m}$. First we see that $c_{1}>-\infty$. From the intersection assumption (4) and the subadditivity property we have that, for $1 \leq l \leq m, A \in \mathscr{A}_{l}$ implies $A=h(S) \backslash K$ and $\gamma(\overline{A \cap Y})=\gamma\left(\overline{\mathscr{S}_{h} \backslash K}\right) \geq l$. This and assumption (2) yield $c_{1}>-\infty$. It is also clear that $c_{m}<0$, since we can take $S \in \mathscr{A}_{m}$. In order to show that the numbers $c_{l}$ are critical values we proceed from here in the standard way. Only note that the Deformation Lemma will provide an odd homeomorphism $\eta: E \rightarrow E$ and if $h \in \Gamma$, then $\eta \circ h$ also belongs to $\Gamma$. See [7].

In what follows we will prove that in our situation we can use Theorem 3.1. For a small number $\varepsilon>0$, to be fixed later, we choose $\psi_{1}^{\varepsilon}, \ldots, \psi_{k}^{\varepsilon}$ as in Lemma 1.1, part (a) and set

$$
V_{1}=\operatorname{span}\left\{\psi_{1}^{\varepsilon}, \ldots, \psi_{k}^{\varepsilon}\right\} \text { and } V_{2}=\operatorname{span}\left\{\psi_{k+1}, \ldots, \psi_{s}\right\}
$$

Also we define

$$
Y=\left\{y \in X \mid \int_{\Omega} \nabla y \nabla \phi_{i}=0, i=1, \ldots, k\right\} .
$$

Then, by construction, we have that $V_{1} \cap Y=\{0\}$, and hence $X=V_{1} \oplus Y$. For small $\varepsilon>0$ we also have $V_{1} \cap V_{2}=\{0\}$.

Lemma 3.2. The functional $J_{\lambda}$ defined in (0.5) satisfies

(1) $J_{\lambda}$ is bounded below in $Y$, and

(2) There exist numbers $b, \rho>0$ such that

$$
J_{\lambda}(w) \leq-b \quad \forall w \in V_{1} \oplus V_{2} \text { with }\|w\|_{X}=\rho .
$$

Proof. In order to prove (1) it suffices to verify that

$$
J_{\lambda}(u) \rightarrow+\infty \text { if }\|u\|_{H_{0}^{\prime}} \rightarrow+\infty, u \in Y .
$$

Assume the contrary, that is, the existence of $c>0$ and a sequence $\left\{u_{n}\right\} \subset Y$ with $\left\|u_{n}\right\|_{H_{0}^{1}} \rightarrow+\infty$ so that $J_{\lambda}\left(u_{n}\right) \leq c$, namely

$$
\frac{1}{2} \int_{\Omega}\left|\nabla u_{n}\right|^{2}-\lambda u_{n}^{2}+\frac{1}{p+1} \int_{\Omega} h\left|u_{n}\right|^{p+1} \leq c .
$$

We observe that $\left\|u_{n}\right\|_{L^{2}} \rightarrow+\infty$ and if we let $\hat{u}_{n}=u_{n} /\left\|u_{n}\right\|_{L^{2}}$, then

$$
\frac{1}{2} \int_{\Omega}\left(\left|\nabla \hat{u}_{n}\right|^{2}-\lambda\right)+\left\|u_{n}\right\|_{L^{2}}^{p-1}\left(\frac{1}{p+1} \int_{\Omega} h\left|\hat{u}_{n}\right|^{p+1}\right) \leq \frac{c}{\left\|u_{n}\right\|_{L^{2}}^{2}} .
$$


Hence $\hat{u}_{n}$ is bounded in $H_{0}^{1}(\Omega)$ and we may assume that $\hat{u}_{n} \rightarrow \hat{u}$ weakly in $H_{0}^{1}(\Omega)$ and strongly in $L^{2}(\Omega)$. From (3.4), using Fatou's Lemma we obtain $\int_{\Omega} h|\hat{u}|^{p+1}=0$; hence $\hat{u} \in H_{*}^{1}\left(\Omega_{0}\right)$. But $\hat{u}$ also belongs to $Y$, the $H_{0}^{1}$ orthogonal of $\operatorname{span}\left\{\phi_{1}, \ldots, \phi_{k}\right\}$, hence to the $H_{*}^{1}$-orthogonal of this space. On the other hand (3.4) also yields

$$
\int_{\Omega}|\nabla \hat{u}|^{2} \leq \lambda
$$

which, by the variational characterization of the eigenvalues, implies $\lambda \geq$ $\lambda_{k+1}\left(\Omega_{0}\right)$. This contradicts our hypothesis (3.1) and (1) is therefore proved.

Next we prove (2). Let

$$
w=\sum_{i=1}^{s} t_{i}\left(\psi_{i}+\eta_{i}^{\varepsilon}\right)
$$

where $\eta_{i}^{\varepsilon}=\psi_{i}^{\varepsilon}-\psi_{i}$ if $i=1, \ldots, k$ and $\eta_{i}^{\varepsilon}=0$ if $i=k+1, \ldots, s$. We compute

$$
\begin{aligned}
J_{\lambda}(w)= & \frac{1}{2} \sum_{i=1}^{s} t_{i}^{2}\left(\lambda_{i}(\Omega)-\lambda\right)+\frac{1}{p+1} \int_{\Omega} h|w|^{p+1} \\
& +\frac{1}{2} \sum_{i=1}^{s} \sum_{j=1}^{s} t_{i} t_{j}\left\{\int_{\Omega} \nabla\left(\psi_{i}+\eta_{i}^{\varepsilon}\right) \nabla \eta_{j}^{\varepsilon}-\lambda\left(\psi_{i}+\eta_{i}^{\varepsilon}\right) \eta_{j}^{\varepsilon}\right\} .
\end{aligned}
$$

Hence, letting $|w|^{2}=\sum_{i=1}^{s} t_{i}^{2}$ we obtain

$$
J_{\lambda}(w) \leq-c_{1}|w|^{2}+c_{2} \varepsilon|w|^{2}+c_{3}|w|^{p+1}
$$

for certain positive constants $c_{1}, c_{2}$ and $c_{3}$, independent of $\varepsilon$. From (3.7), the result of (2) readily follows after choosing $\varepsilon$ sufficiently small.

In the rest of this section we will denote

$$
S_{\rho}=\left\{w \in V_{1} \oplus V_{2} \mid\|w\|_{X}=\rho\right\}
$$

where $\rho$ is chosen as in Lemma 3.2. First we define the class of functions

$$
\Gamma=\left\{h: S_{\rho} \rightarrow X \backslash\{0\} \mid h \text { is continuous and odd }\right\} \text {. }
$$

We note that if $\eta: X \rightarrow X$ is an odd homeomorphism, then $\eta \circ h \in \Gamma$ for all $h \in \Gamma$.

The next lemma gives an intersection result that implies hypothesis (4) in Theorem 3.1.

Lemma 3.3. For $h \in \Gamma$ we let $\mathscr{S}^{h}=h\left(S_{\rho}\right) \cap Y$. Then $\gamma\left(\mathscr{S}^{h}\right) \geq s-k$.

Proof. Since $S_{\rho} \subset V_{1} \oplus V_{2}$ is finite dimensional, $A=h\left(S_{\rho}\right)$ is compact. By the dimension property of the genus, $\gamma(A)=S$. Then Lemma 3.1 gives the result.

Proof of Theorem 0.2. Lemmas 3.2 and 3.3 imply that Theorem 3.1 is applicable to the functional $J_{\lambda}$ when $\lambda$ satisfies $(0.4)$. Thus there exist pairs of solutions $\pm u_{\lambda}^{k+l}, l=1, \ldots, s-k$, such that

$$
J_{\lambda}\left(u_{\lambda}^{k+l}\right)=c_{l}(\lambda)=\inf _{A \in \mathscr{S}_{l}} \sup _{u \in A} J_{\lambda}(u), \quad l=1, \ldots, s-k,
$$


where the class of sets $\mathscr{A}_{l}$ was defined in the proof of Theorem 3.1. Let $q$ be the multiplicity of $\lambda_{k}\left(\Omega_{0}\right)$ and denote $\bar{q}=\min \{q, s-k\}$. It is easily checked that the validity of assertions (i) and (ii) of Theorem 0.2 will follow if we prove the following two facts:

Claim 1. $c_{\bar{q}}(\lambda) \rightarrow-\infty$ as $\lambda \rightarrow \lambda_{k+1}\left(\Omega_{0}\right)$.

Claim 2. If $l$ satisfies $q<l \leq s-k$, then there is a real number $c$ such that $c_{l}(\lambda) \geq c$ for all $\lambda$ satisfying $(0.4)$.

For the proof of Claim 1, we need some estimates for $J_{\lambda}$ which we carry out next. In the rest of the proof we will assume $\bar{q}=q<s-k$, the other case being similar.

Note that the functions $\phi_{k+1}, \ldots, \phi_{k+q}$ are not solutions of the equation

$$
\begin{aligned}
-\Delta u & =\lambda_{k+1}\left(\Omega_{0}\right) u \text { in } \Omega, \\
u & =0 \text { on } \partial \Omega ;
\end{aligned}
$$

hence the functionals $f_{i} \in H^{-1}(\Omega), i=1, \ldots, q$, defined as

$$
f_{i}(\varphi)=\int_{\Omega} \nabla \phi_{k+i} \nabla \varphi-\lambda_{k+1}\left(\Omega_{0}\right) \phi_{k+i} \varphi \quad \forall \varphi \in H_{0}^{1}(\Omega)
$$

are linearly independent. This implies the existence of functions $v_{1}, \ldots, v_{q} \in$ $H_{0}^{1}(\Omega)$ so that the matrix $A=\left\{a_{i j}\right\}$ defined as

$$
a_{i j}=f_{i}\left(v_{j}\right), \quad 1 \leq i, j \leq q,
$$

is invertible. Moreover, we may assume without loss of generality that the functions $v_{j}$ satisfy

$$
\int_{\Omega} \nabla \phi_{l} \nabla v_{j}=0, \quad 1 \leq j \leq q
$$

and $1 \leq l \leq k, k+q+1 \leq l \leq s$.

Let us consider $w_{1} \in \operatorname{span}\left\{\phi_{1}, \ldots, \phi_{k}\right\}$ and $w_{2} \in \operatorname{span}\left\{\phi_{k+q+1}, \ldots, \phi_{s}\right\}$. For $\theta, t \in \mathbb{R}^{q}$ with components $\theta_{i}$ and $t_{i}, 1 \leq i \leq q$, define $v=\sum_{i=1}^{q} \theta_{i} v_{i}$ and $u=\sum_{i=1}^{q} t_{i} \phi_{i+k}$. We want to estimate $J_{\lambda}\left(w_{1}+v+u+w_{2}\right)$. Using the definition of $J_{\lambda}$ we find that

$$
\mathscr{J} \equiv J_{\lambda}\left(w_{1}+v+u+w_{2}\right)=\mathscr{J}_{1}+\mathscr{J}_{2}
$$

where

$$
\mathscr{J}_{1}=J_{\lambda}\left(w_{1}\right)+J_{\lambda}\left(w_{2}\right)+J_{\lambda}(v)-\lambda \int_{\Omega}\left(w_{1}+w_{2}\right) v
$$

and

$$
\mathscr{J}_{2}=\frac{\lambda_{k+1}\left(\Omega_{0}\right)-\lambda}{2}|t|^{2}+\sum_{i=1}^{q} \sum_{j=1}^{q} \theta_{i} t_{j} \int_{\Omega} \nabla v_{i} \nabla \phi_{j+k}-\lambda v_{i} \phi_{j+k}
$$

Using the matrix $A$ defined in (3.11) we rewrite this last term as

$$
\mathscr{J}_{2}=\frac{\lambda_{k+1}\left(\Omega_{0}\right)-\lambda}{2}|t|^{2}+\left(\lambda_{k+1}\left(\Omega_{0}\right)-\lambda\right) \sum_{i=1}^{q} \sum_{j=1}^{q} \theta_{i} t_{j} \int_{\Omega} v_{i} \phi_{j+k}+\left(\theta, A^{\tau} t\right)
$$


where $|\cdot|$ and $(\cdot, \cdot)$ denote the usual norm and inner product in $\mathbb{R}^{q}$, and $A^{\tau}$ represents the transpose of $A$. Then, assuming that $\theta$ is bounded, from (3.16) we obtain

$$
\mathscr{J}_{2} \leq \frac{\lambda_{k+1}\left(\Omega_{0}\right)-\lambda}{2}\left(|t|^{2}+a_{1}|t|\right)+\left(\theta, A^{\tau} t\right) .
$$

Here and henceforth we use the notation $a_{1}, a_{2}$, etc., to designate different positive absolute constants. On the other hand, assuming that $\theta$ is bounded, from (3.14) we have that

$$
\mathscr{J}_{1} \leq \frac{\lambda_{k}\left(\Omega_{0}\right)-\lambda}{2}\left\|w_{1}\right\|_{L^{2}}^{2}+a_{1}\left\|w_{2}\right\|_{L^{2}}^{2}+a_{2}\left\|w_{1}+w_{2}\right\|_{L^{2}}+a_{3} .
$$

Next we will find an appropriate function $h$ in $\Gamma$ and a set $K$ with $\gamma(K) \geq$ $(s-k)-q$ in order to estimate the critical value $c_{q}(\lambda)$ when $\lambda$ is close to $\lambda_{k+1}\left(\Omega_{0}\right)$. Let us fix a number $\sigma \geq 1$. We define the function $h_{\sigma}: V_{1} \oplus V_{2} \rightarrow X$ in the following manner. For $\alpha_{i} \in \mathbb{R}, 1 \leq i \leq s$, we put

$$
v_{1}=\sum_{i=1}^{k} \alpha_{i} \psi_{i}^{\varepsilon}, \quad \bar{v}=\sum_{i=k+1}^{k+q} \alpha_{i} \psi_{i}, \quad v_{2}=\sum_{i=k+q+1}^{s} \alpha_{i} \psi_{i}
$$

and define

$$
h_{\sigma}\left(v_{1}+\bar{v}+v_{2}\right)=\sigma^{1 / 2} w_{1}+v+\sigma u+w_{2}
$$

where

$$
w_{1}=\sum_{i=1}^{k} \alpha_{i} \phi_{i}, \quad v=\sum_{i=k+1}^{k+q} \beta_{i} v_{i-k}, \quad u=\sum_{i=k+1}^{k+q} \alpha_{i} \phi_{i}, \quad w_{2}=\sum_{i=k+q+1}^{s} \alpha_{i} \phi_{i},
$$

and the coefficients $\beta_{i}$ are given by

$$
\beta_{i}=-\sum_{j=1}^{q} a_{j i} \alpha_{j}
$$

Next define, for a small $\nu>0$ and $h_{\sigma}$ as in (3.19), the compact set

$$
K_{\nu}=\left\{h_{\sigma}\left(v_{1}+\bar{v}+v_{2}\right)\left\|v_{1}+\bar{v}+v_{2}\right\|_{X}=\rho,\left\|v_{2}\right\|_{X} \geq \nu\right\} .
$$

It is easy to see that $\gamma\left(K_{\nu}\right)=\gamma\left(K_{1}\right)$ and then $\gamma\left(K_{\nu}\right)=s-(k+q)=(s-k)-q$. On the other hand, for $z \in h_{\sigma}\left(S_{\rho}\right) \backslash K_{\nu}$, equations (3.17), (3.18) and (3.20) imply that

$$
\begin{aligned}
J_{\lambda}(z) \leq & \left(\lambda_{k+1}\left(\Omega_{0}\right)-\lambda\right) a_{1} \sigma^{2}-\sigma a_{2}\|\bar{v}\|_{X}^{2} \\
& +\left(\lambda_{k}\left(\Omega_{0}\right)-\lambda\right) \sigma\left\|v_{1}\right\|_{L^{2}}^{2}+\sigma^{1 / 2} a_{3}+a_{4} .
\end{aligned}
$$

If $\|\bar{v}\|_{X} \geq \rho / 4$, from (3.22) we find that

$$
J_{\lambda}(z) \leq\left(\lambda_{k+1}\left(\Omega_{0}\right)-\lambda\right) a_{1} \sigma^{2}-\sigma \rho a_{2}+a_{3} \sigma^{1 / 2}+a_{4}
$$

and if $\|\bar{v}\|_{X}<\rho / 4, \lambda \geq \tilde{\lambda}>\lambda_{k}\left(\Omega_{0}\right)$ and $\nu<\rho / 4$, we have from (3.22) that

$$
J_{\lambda}(z) \leq\left(\lambda_{k+1}\left(\Omega_{0}\right)-\lambda\right) a_{1} \sigma^{2}-a_{2} \sigma \rho^{2}+a_{3} \sigma^{1 / 2}+a_{4} .
$$

Now, combining (3.23) and (3.24) we observe that an appropriate choice of $\sigma$ will yield

$$
J_{\lambda}(z) \leq-\frac{b_{1}}{\lambda_{k+1}\left(\Omega_{0}\right)-\lambda}+b_{2} \quad \forall z \in h_{\sigma}\left(S_{\rho}\right) \backslash K_{\nu}
$$


for $\lambda$ close to $\lambda_{k+1}\left(\Omega_{0}\right)$, where the positive constants $b_{1}$ and $b_{2}$ may depend on $\rho$ put not on $\lambda$. The definition of $c_{q}(\lambda)$ as a minimax implies that

$$
c_{q}(\lambda) \leq \sup \left\{J_{\lambda}(z) \mid z \in h_{\sigma}\left(S_{\rho}\right) \backslash K_{\nu}\right\}
$$

from which the validity of Claim 1 follows at once.

Next we prove Claim 2. If we define

$$
Y_{q}=\left\{y \in X \mid \int_{\Omega} \nabla y \nabla \phi_{i}=0,1 \leq i \leq k+q\right\},
$$

then the same argument given for the proof of Lemma 3.3 will imply that

$$
\gamma\left(\mathscr{S}^{h, q}\right) \geq s-(k+q) \quad \forall h \in \Gamma
$$

where $\mathscr{S}^{h, q}=h\left(S_{\rho}\right) \cap Y_{q}$. Consequently, for $l \geq q+1$ we will have that

$$
\gamma\left(\overline{h\left(S_{\rho}\right) \backslash K \cap Y_{q}}\right) \geq l-q \geq 1 \text {. }
$$

Thus, for every $A \in \mathscr{A}_{l}$ there is $y \in A$ such that $y \in Y_{q}$. Following the estimates that led us to Lemma 3.2, part (1) we will find that $J_{\lambda}$ is bounded below by a constant independent of $\lambda$, for $\lambda$ satisfying (0.4). Thus $c_{l}(\lambda) \geq c$ for all $q+1 \leq l \leq s$ if $\lambda \leq \lambda_{k+1}\left(\Omega_{0}\right)$, as desired. Thus Claim 2 is established, and the theorem follows.

Remark 3.1. It should be noticed that the above proof does not require homogeneity of the nonlinearity, and will therefore apply to the more general problem (1.14)-(1.15) as long as $f$ is odd, increasing and satisfies appropriate polynomial growth restrictions near 0 and $\infty$.

\section{REFERENCES}

1. V. Benci, On critical point theory for indefinite functionals in the presence of symmetries, Trans. Amer. Math. Soc. 274 (1982), 533-572.

2. D. Clark, A variant of the Lusternik-Schnirelman theory, Indiana Univ. Math. J. 22 (1972), 65-74.

3. M. del Pino, Positive solutions of a semilinear elliptic equation on a compact manifold (to appear).

4. J. Kazdan and F. Warner, Scalar curvature and conformal deformation of Riemannian structure, J. Differential Geom. 10 (1975), 113-134.

5. T. Ouyang, On the positive solutions of semilinear equations $\Delta u+\lambda u-h u^{p}=0$ on compact manifolds, Trans. Amer. Math. Soc. 331 (1992), 503-527.

6. - On the positive solutions of semilinear equations $\Delta u+\lambda u-h u^{p}=0$ on compact manifolds, Part II, Indiana Univ. Math. J. 40 (1991), 1083-1140.

7. P. Rabinowitz, Minimax methods in critical point theory with applications to differential equations, C.B.M.S. Regional Conf. Ser. in Math., no. 65, American Mathematical Society, Providence, RI, 1986.

Department of Mathematics, University of Chicago, Chicago, Illinois 60637

E-mail address: delpinomath.uchicago.edu

Departamento de Ingenieria Matemática, F.C.F.M., Universidad de Chile, Casilla 170 Correo 3, Santiago, Chile

E-mail address: pfelmer@llaima.dim.uchile.cl 